\title{
Phaeochromocytoma of the urinary bladder
}

\author{
Sarmah PB, Kelly BD*, Noah A and Ryan PG \\ Department of Urology, City Hospital, Birmingham, UK
}

\begin{abstract}
Phaeochromocytoma is a benign tumour which mostly occurs in the adrenal glands but can arise in extra-adrenal locations. We report a case in a Caucasian male who presented only with visible haematuria. Flexible cystoscopy revealed a mass on the left lateral wall, confirmed on a contrast-enhanced computed tomography scan of the urinary tract. The patient underwent a transurethral resection of bladder tumour with histological analysis demonstrating paraganglioma. Further reresection demonstrated a small nodule of recurrent disease in the lamina propria. Phaeochromocytoma of the urinary bladder is a rare condition which requires close investigation and follow-up due to its malignant potential.
\end{abstract}

\section{Introduction}

Phaeochromocytoma commonly affects the adrenal gland but can have extra-adrenal locations due to distribution along the paraganglion system, including the urinary bladder. We report a case in a male patient without typical symptoms, and discuss the presentation, diagnosis and management of this condition.

\section{Clinical Practice Points}

A 56 year old male was seen in the Urology two week wait clinic with visible haematuria. He had undergone a flexible cystoscopy in his native country one month earlier and been informed that he had a bladder mass, however no biopsy was taken as he was taking Warfarin at the time for atrial fibrillation and he was advised to return to the UK. He had a further background history of Type II diabetes and hypertension, and was taking medications in the form of insulin, metformin, amlodipine, bisoprolol, ramipril and torasemide. He was a non-smoker and unemployed. On examination he was markedly obese. Abdominal examination revealed no tenderness or abdominal masses. There was a buried penis. Flexible cystoscopy was performed which demonstrated a large $3 \times 4 \mathrm{~cm}$ solid mass on the left lateral wall. Blood tests revealed impaired renal function but normal electrolytes (Table 1) and full blood count, and urine cytology and culture were normal. A computed tomography (CT) scan of the urinary tract with contrast was performed confirming the presence of a solid mass against the left lateral wall of the bladder with minor perivesical stranding (Figure 1), but normal upper urinary tracts.

The patient underwent a transurethral resection of bladder tumour (TURBT) with the findings from flexible cystoscopy confirmed, and post-operatively had instillation of intravesical Mitomycin-C. Histology from the operative chippings included muscularis propria and found solid nests of cells diffusely arranged with a slightly packeted arrangement, separate by prominent vasculature. The tumour cells were immunoreactive for S100 and CD56, and overall appearances were consistent with an extra adrenal paraganglioma or phaeochromocytoma. A repeat TURBT and random bladder biopsy was performed two months later which confirmed a small nodule of recurrent extra adrenal phaeochromocytoma, located mainly in the lamina propria. A methyliodobenzylguanidine (MIBG) scintigram
Table 1. Blood results on admission

\begin{tabular}{|c|c|}
\hline \multicolumn{2}{|c|}{ Urea and electrolytes } \\
\hline Sodium & $135 \mathrm{mmol} / \mathrm{L}$ \\
\hline Potassium & $4.9 \mathrm{mmol} / \mathrm{L}$ \\
\hline Urea & $18.2 \mathrm{mmol} / \mathrm{L}$ \\
\hline Creatinine & $165 \mathrm{umol} / \mathrm{L}$ \\
\hline Estimated glomerular filtration rate & $45 \mathrm{~mL} / \mathrm{min} / 1.73 \mathrm{~m}^{2}$ \\
\hline
\end{tabular}

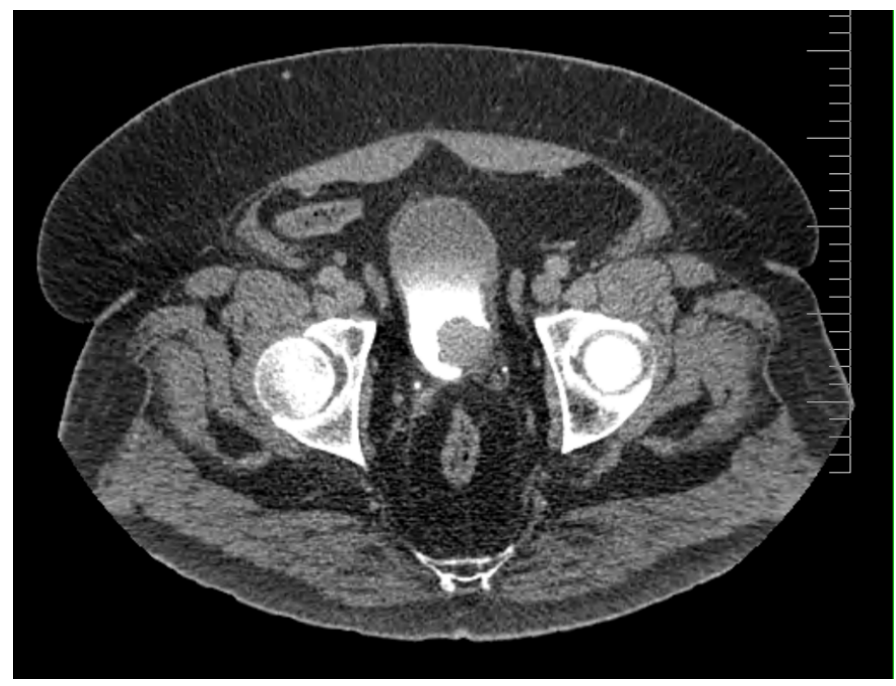

Figure 1. Axial section CT scan of the urinary tract demonstrating solid mass arising from left lateral wall of the urinary bladder

demonstrated no concurrent disease or residual disease in the wall of the bladder. The case was discussed at multi-disciplinary conference where the outcome was for a further re-resection in three months. The patient has subsequently undergone 2 years of surveillance but with no evidence of recurrence.

${ }^{*}$ Correspondence to: Sarmah PB, Department of Urology, City Hospital, Birmingham, UK, E-mail: piyushbsarmah@doctors.org.uk

Received: April 23, 2020; Accepted: May 06, 2020; Published: May 11, 2020 


\section{Discussion}

Phaeochromocytoma is a catecholamine-producing tumour derived from chromaffin cells, most commonly in the adrenal glands. However extra-adrenal occurrences have been described in the literature and can account for up to $15 \%$ of all adult cases [1]. This includes the urinary tract with the urinary bladder acting as the most common site, although in total phaeochromocytoma makes up less than $0.05 \%$ of all bladder tumours [2]. The commonly recognised signs and symptoms from excess of circulating catecholamines can occur in association with micturition, for example palpitations, hypertension, headache and flushing [3]. Haematuria, as for other bladder tumours, is also a common presentation in just under $50 \%$ of patients as it was in this case [2]. This was enough to warrant urgent investigation in the form of direct cystoscopic visualisation. Even so, phaeochromocytoma was only detected after histological analysis and was a surprising diagnosis in the absence of other symptoms as mentioned above. This emphasises the diagnostic challenge faced with phaeochromocytoma especially in the absence of typical clinical features of the disease.

Pre-operatively diagnosis can be aided with measurement of serum and urinary catecholamines and their metabolites (metanephrine and vanillyl mandelic acid). This condition should ideally be detected preoperatively to avoid hypertensive crises at the time of operation via excess manipulation of the tumour. Transurethral resection of bladder tumour (TURBT) has been advocated as a suitable surgical option by Ahn, et al. for small, minimally invasive, non-metastatic, hormonally inactive tumours [4]. It is however still necessary to obtain histological confirmation of the disease.

Different imaging modalities are used for extra-adrenal phaechromocytoma. Given that $10 \%$ of phaeochromocytomas have malignant potential, [5] both local and distant staging of the tumour are performed with contrast-enhanced computed tomograghy (CT) and magnetic resonance imaging (MRI) the most commonly used investigations; the latter is preferable specifically for imaging the pelvis as the detection rate for submucosal and smaller tumours is greater [6]. However the upper urinary tracts still warrant imaging in investigation of haematuria as happened in this case. Functional activity can be confirmed with the use of methyliodobenzylguanidine (MIBG) scintigraphy, which may be performed either as an adjunct if phaeochromocytoma has already been diagnosed histologically or to distinguish bladder masses on CT or MRI which are thought to be submucosal in nature [6]. The most commonly used radioisotope was $\mathrm{I}^{131}$ although improved visualisation has been demonstrated with I [13] instead [7].
Many treatment modalities exist for phaeochromocytoma of the bladder. Surgery in the form of either partial or radical cystectomy has been demonstrated as the preferred option in $70-80 \%$ of cases, most likely performed in order to achieve a greater degree of clearance with these submucosal tumours which have a greater malignant potential [6]. This has traditionally been performed via open repair but more recently laparoscopic and robot-assisted operations have been reported $[8,9]$. In this case the tumour was histologically non-invasive, and due to the patient's obese state repeat TURBT was considered to be a safer option as he would have been high risk for major surgery. Regardless of the surgical intervention performed, lifelong follow-up and monitoring of disease recurrence is mandatory by measuring catecholamine levels owing to the potentially malignant nature of the condition [10].

\section{Conclusion}

Phaeochromocytoma of the urinary bladder is a rare condition which requires close investigation and follow-up due to its malignant potential.

\section{References}

1. Whalen RK, Althausen AF, Daniels GH (1992) Extra-adrenal pheochromocytoma. $J$ Urol 147: 1-10. [Crossref]

2. Beilan JA, Lawton A, Hajdenberg JRC (2013) Pheochromocytoma of the urinary bladder: a systematic review of the contemporary literature. Biomed Cent Urol p. 13.

3. Naqiyah I, Rohaizak M, Meah FA, Nazri MJ, Sundram M, et al. (2005) Phaeochromocytoma of the urinary bladder. Singapore Med J 46: 344-346. [Crossref]

4. Ahn SG, Jang H, Han DS (2013) Transurethral resection of bladder tumour (TURBT) as an optional treatment method on pheochromocytoma of the urinary bladder. $J$ Can Urol Assoc 7: 130-134

5. Rohan V, Tankshali R, Hanji A (2012) Pheochromocytoma of the urinary bladder: A rare cause of severe hypertension. Saudi J Kidney Dis Transplant 23: 813.

6. Tsai CC, Wu WJ, Chueh KS (2011) Paraganglioma of the urinary bladder first presented by bladder bloody tamponade: Two case reports and review of the literatures. Kaohsiung J Med Sci 27:108-113.

7. Nakatani T, Hayama T, Uchida J (2002) Diagnostic localization of extra-adrenal pheochromocytoma: comparison of (123) I-MIBG imaging and (131) I-MIBG imaging. Oncol Rep 9: 1225-1227.

8. Huang Y, Tian XJ ML (2009) Pre-peritoneal laparoscopic partial cystectomy of the bladder pheochromocytoma. Chinese Med J 122: 1234-1237.

9. Kang SG, Kang SH, Choi H (2011) Robot-assisted partial cystectomy of a bladder pheochromocytoma. Urol Int 87: 241-244.

10. Li W, Yang B, Che JP (2013) Diagnosis and treatment of extra-adrena pheochromocytoma of urinary bladder: case report and literature review. Int $J$ Clin Exp Med 6: 832-829.

Copyright: (C2020 Sarmah PB. This is an open-access article distributed under the terms of the Creative Commons Attribution License, which permits unrestricted use, distribution, and reproduction in any medium, provided the original author and source are credited. 